\title{
Aspectos clínicos e patogenéticos da intoxicação por abamectina em bezerros ${ }^{1}$
}

\author{
Josilene N. Seixas ${ }^{2 *}$, Paulo V. Peixoto ${ }^{3}$, Anibal G. Armién ${ }^{4}$, Flávia F. Jabour ${ }^{5}$ e \\ Marilene F. Brito 6
}

\begin{abstract}
Seixas J.N., Peixoto P.V., Armién A.G., Jabour F.F. \& Brito M.F. 2006. [Clinical and pathogenetic aspects of abamectin poisoning in calves.] Aspectos clínicos e patogenéticos da intoxicação por abamectina em bezerros. Pesquisa Veterinária Brasileira 26(3):161-166. Projeto Sanidade Animal Embrapa/UFRRJ, Km 47, Seropédica, RJ 23890-000, Brazil. E-mail: josiseixas@ufrrj.br

Clinic-pathological aspects and the pathogenesis of experimental abamectin poisoning were studied, after subcutaneous administration of different abamectin doses in 9 calves, as well as the clinical and pathological aspects of 74 cases of the iatrogenic poisoning with this drug in cattle, which occurred in the states of Rio Grande do Sul, Pará, Maranhão, Paraíba and Mato Grosso do Sul. From the 9 calves submitted to experimental administration of single doses, 5 calves died 4 calves received doses 6-10 times higher than recommended, and one received the therapeutic daily dose during 11 days). Abamectin poisoning induces neurological dysfunctions, characterized by an initial phase of hyperexcitability, followed by widespread muscular hypotony and progressive depression. No macroscopic or microscopic alterations were observed in the central nervous system or in any other organ. It is concluded that abamectin is an antihelmintic which should be used with restriction, because of the risks leading to death when used in young calves, even in therapeutic doses.
\end{abstract}

INDEX TERMS: Abamectin poisoning, neurological alterations, calves.

RESUMO.- Estudaram-se os aspectos clínico-patológicos e patogenéticos verificados após a administração experimental subcutânea de diferentes doses de abamectina em nove bezerros. Também são apresentados os dados clínico-patológicos sobre a ocorrência de intoxicação iatrogênica por essa droga que resultaram em 74 mortes em bovinos nos Estados do Rio Grande do Sul, Pará, Maranhão, Paraíba e Mato Grosso do Sul. No presente estudo, dos nove bezerros submetidos à administração experimental, cinco morreram (quatro que receberam doses únicas de 6-10 vezes superior à recomendada e um que recebeu diariamente a dose terapêutica durante 11 dias). A intoxicação

\footnotetext{
${ }^{1}$ Recebido em 7 de fevereiro de 2006.

Aceito para publicação em 22 de fevereiro de 2006.

${ }^{2}$ Parte da dissertação de mestrado no curso de Medicina Veterinária da Universidade Federal Rural do Rio de Janeiro (UFRRJ). "Autor para correspondência: josiseixas@ufrrj.br

${ }^{3}$ Depto Nutrição Animal e Pastagem, Instituto de Zootecnia, UFRRJ, Seropédica, RJ 23890-000.

${ }^{4}$ Dept Pathobiological Sciences, University of Wisconsin, Madison, WI 537061102 , USA.

${ }^{5}$ Projeto Sanidade Animal Embrapa/UFRRJ, Seropédica, RJ 23890-000.

${ }^{6}$ Disciplina de Anatomia Patológica, Depto Epidemiologia e Saúde Pública, Instituto de Veterinária, UFRRJ.
}

por abamectina induz a disfunções neurológicas, caracterizadas por uma fase inicial de hiperexcitabilidade, seguida por hipotonia muscular generalizada e depressão progressiva. Nenhuma alteração macroscópica ou microscópica foi observada no sistema nervoso central ou em qualquer outro órgão. Conclui-se que a abamectina é um medicamento que deve ser utilizado com restrições, pois há riscos de morte quando utilizado em bezerros jovens, até mesmo na dose terapêutica.

TERMOS DE INDEXAÇÃO: Intoxicação por abamectina, alterações neurológicas, bezerros.

\section{INTRODUÇÃO}

As avermectinas são metabólitos derivados da fermentação de Streptomyces avermitilis (Burg et al. 1979). Nesse grupo encontram-se a ivermectina, a abamectina e a doramectina, entre outras drogas quimicamente relacionadas e usadas na medicina veterinária e humana, como antiparasitários, e na agricultura, como inseticidas (Barragry 1994, Lankas et al. 1997).

A partir da década de 1980, as avermectinas começaram a ser utilizadas como anti-helmínticos (Taylor 2001, Ayres \& Almeida 2002). A ivermectina, derivado sintético da abamectina, foi a primeira a ser comercializada (Chabala et al. 1980, Steel 1993). Em 1985, a abamectina foi lançada como antiparasitário e inse- 
ticida (Basílio 2005). Atualmente, as avermectinas estão entre as principais drogas utilizadas para o controle de endo e ectoparasitas em animais de produção (Corwin 1999) e a grande conquista do mercado consumidor se deve, em especial, ao amplo espectro de ação e margem de segurança (Forbes 1993).

As avermectinas, em geral, não causam efeitos tóxicos nos animais, pois apresentam alto peso molecular e não atravessam facilmente a barreira hematoencefálica (BHE) para atuarem no sistema nervoso central (SNC) (Lankas \& Gordon 1989, Courtney \& Roberson 1995, Ayres \& Almeida 2002). Qualquer espécie, porém, pode ser afetada se a dose for grande o suficiente para penetrar a BHE (Andrade \& Santarém 2002) e em casos de intoxicação, há histórico de exposição acidental ou contrária às recomendações (Moore 1999). Estes medicamentos também são contra-indicados em casos de doenças que causam danos à BHE (Nobmann et al. 2001, Tracy \& Webster 2001) e devem ser utilizados com cautela em pacientes submetidos a terapias com agentes depressores do SNC (Tracy \& Webster 2001). Algumas raças de cães pastores, bezerros com menos de quatro meses, roedores e coelhos gestantes apresentam uma maior susceptibilidade a fármacos desse grupo (Lankas \& Gordon 1989, Andrade \& Santarém 2002, Ayres \& Almeida 2002).

A droga mais bem caracterizada do grupo, a ivermectina, pode induzir neurotoxicidade e alteração fetal. Surtos ocasionais de alterações neurológicas ocorrem em bovinos da raça Murray Grey que a recebem na dose prescrita, talvez por apresentarem uma maior permeabilidade da barreira BHE (Radostits et al. 2002). A ivermectina não é recomendada para bezerros com menos de 4 meses, dado o desenvolvimento incompleto da BHE (Pulliam \& Preston 1989, Courtney \& Roberson 1995, Ayres \& Almeida 2002). A deficiência de glicoproteína-P no cérebro (Ayres \& Almeida 2002), possivelmente como resultado de um defeito genético autossômico recessivo (Lankas \& Gordon 1989, Andrade \& Santarém 2002, Radostits et al. 2002) reduz o efluxo deste medicamento do SNC (Ayres \& Almeida 2002) e aumenta a susceptibilidade de certos animais à intoxicação; algumas raças de cães como Collie, Old English Sheepdog, Shetland Sheepdogs, Australian Sheepdogs e seus mestiços, bem como cães e gatos com idade inferior a 6 semanas também são mais susceptíveis (Andrade \& Santarém 2002).

Experimentos com intoxicações aguda, subaguda e crônica, realizados com a abamectina em ratos, coelhos, cães e macacos demonstraram resultados similares aos da ivermectina, exceto pela maior toxidez desse fármaco. Em geral, entre as principais manifestações clínicas observaram-se midríase, vômitos, tremores, ataxia, depressão, convulsões, coma e morte, com algumas variações entre as espécies (Lankas \& Gordon 1989).

Pulliam \& Preston (1989) citam os diversos experimentos conduzidos por Button et al. (1988) para avaliar a tolerância de bovinos e búfalos (477 animais) à abamectina através da administração de doses que variaram de $0,3-8 \mathrm{mg} / \mathrm{kg}$. Após a indução da intoxicação aguda, os bovinos apresentaram como primeiros sinais, depressão e ataxia. Sinais moderados de intoxicação foram observados após os bezerros receberem doses a partir de $0,727 \mathrm{mg} / \mathrm{kg}$, aproximadamente 3,6 vezes a dose terapêutica (DT) e a dose máxima tolerada foi de $1 \mathrm{mg} / \mathrm{kg}, 5$ vezes a DT. Quando utilizada em altas doses (2-8mg/kg, ou seja, de $10-40$ vezes a DT) ocorreram severos sinais de toxicidade como ataxia, paresia progressiva, midríase, coma, decúbito, diminuição do tônus da língua e lábios, sialorréia e morte. Nos animais supracitados que morreram, nenhuma alteração macro ou microscópica foi evidenciada (Pulliam \& Preston 1989).

Apesar das avermectinas serem descritas como drogas que possuem uma ampla margem de segurança (Lankas \& Gordon 1989, Courtney \& Roberson 1995, Ayres \& Almeida 2002), no Brasil o uso equivocado desses medicamentos em bezerros jovens têm resultado no aparecimento de casos de intoxicação iatrogênica, seguidos ou não de morte. Diante da obtenção de históricos de intoxicação por avermectinas em diferentes localidades do país, foram realizados estudos experimentais através da administração de abamectina a bezerros jovens, com o objetivo de descrever detalhadamente o quadro clínico-patológico, bem como levantar elementos para o estabelecimento da patogenia envolvida.

\section{MATERIAL E MÉTODOS}

\section{Casos naturais}

Dos seguintes locais, foram obtidos históricos sobre bezerros que, após administração de abamectina, evidenciaram sinais de intoxicação: Cruz Alta, RS (Driemeier 2001), Rondon do Pará, PA, Capitão Poço, PA e Açailândia, MA (Barbosa 2004), São Mamede, PB (Riet-Correa 2006), Corumbá, MS e São Nicolau, RS (Wouters 2005).

\section{Casos experimentais}

Os experimentos foram realizados nas dependências do Projeto Sanidade Animal Embrapa/Universidade Federal Rural do Rio de Janeiro, município de Seropédica, Rio de Janeiro.

Abamectina foi administrada por via subcutânea, em doses únicas ou repetidas, a nove bezerros machos, mestiços da raça holandesa, com idades aproximadas de 2-8 semanas.

Antes do início dos experimentos, os bezerros foram colocados em baias individuais; adotou-se o manejo do local de origem (Pesagro, Estação experimental de Seropédica, Área de Bovino de Leite), ou seja, a cada animal eram dados 4 litros de leite in natura, uma vez ao dia, pela manhã e $100 \mathrm{~g}$ de ração. Durante a realização do experimento, os animais foram acompanhados por um período mínimo de uma semana ou até apresentarem-se clinicamente sadios. Exames semiológicos completos foram realizados durante todo o período de permanência dos animais no experimento. $\mathrm{O}$ delineamento experimental encontra-se detalhado no Quadro 1.

Os animais que morreram foram necropsiados de imediato; fragmentos de todos os órgãos foram coletados e fixados em formalinaPBS (fosfato salino tamponado) a $10 \%$ e os do SNC, em formalina-PBS a $20 \%$, por pelo menos 48 horas, processados pelos métodos de rotina, cortados na espessura de 5 micrômetros e as lâminas, coradas pela Hematoxilina-Eosina.

\section{RESULTADOS}

\section{Históricos de casos naturais no Brasil}

No município de Cruz Alta, RS, verificou-se a ocorrência de 6 mortes em bezerros do após administração de abamectina, cuja dose não foi informada pelo proprietário; os bezerros apresentaram apatia, dificuldade locomotora, salivação e morte. Desses animais, um bezerro macho de 10 meses de idade morreu após três dias e foi encaminhado ao Setor de Patologia Veterinária da Universidade Federal do Rio Grande do Sul. (Driemeier 2001) 
Quadro 1. Delineamento experimental e desfecho na intoxicação por abamectina em bezerros

\begin{tabular}{|c|c|c|c|c|c|c|c|c|}
\hline $\begin{array}{c}\text { Animal } \\
\text { no. }\end{array}$ & $\begin{array}{l}\text { Idade } \\
\text { (dias) }\end{array}$ & $\begin{array}{r}\text { Peso } \\
(\mathrm{kg})\end{array}$ & $\begin{array}{c}\text { Numero de } \\
\text { administrações }\end{array}$ & $\begin{array}{c}\text { Dose } \\
\text { terapêutica } \\
(\mathrm{ml}) \\
\end{array}$ & $\begin{array}{l}\text { Dose } \\
\text { diária } \\
(\mathrm{ml}) \\
\end{array}$ & $\begin{array}{c}\text { Início dos sinto- } \\
\text { mas após a } 1^{\underline{a}} \\
\text { administração }\end{array}$ & Evolução & Desfecho \\
\hline 1346 & 15 & 49,0 & 21 & 0,5 & $0,49^{\mathrm{a}}$ & $1 \mathrm{~h}$ & 21 dias & Recuperou-se \\
\hline 1344 & 18 & 47,0 & 11 & 1 & $0,94^{\mathrm{a}}$ & $1 \mathrm{~h}$ & 11dias & Morreu \\
\hline 1340 & 16 & 44,5 & 1 & 1 & $0,89^{b}$ & $4 \mathrm{~h} 45 \mathrm{~min}$ & $99 \mathrm{~h} 34 \mathrm{~min}$ & Recuperou-se \\
\hline 1342 & 16 & 42,5 & 1 & 5 & $4,25^{b}$ & $10 \mathrm{~h} 24 \mathrm{~min}$ & 11 dias & Recuperou-se \\
\hline 1325 & 19 & 40,0 & 1 & 5 & $4,00^{\mathrm{b}}$ & 1h30min & $94 \mathrm{~h} 20 \mathrm{~min}$ & Recuperou-se \\
\hline 1347 & 15 & 41,9 & 1 & 6 & $5,04^{b}$ & $50 \mathrm{~min}$ & $33 \mathrm{~h} 20 \mathrm{~min}$ & Morreu \\
\hline 1343 & 15 & 52,0 & 1 & 7,5 & $7,80^{\mathrm{b}}$ & $2 \mathrm{~h} 20 \mathrm{~min}$ & $20 \mathrm{~h} 50 \mathrm{~min}$ & Morreu \\
\hline 1320 & 20 & 43,0 & 1 & 10 & $8,60^{\mathrm{b}}$ & $12 \mathrm{~h}$ & $13 \mathrm{~h} 45 \mathrm{~min}$ & Morreu \\
\hline 1524 & 56 & 55,0 & 1 & 10 & $11,00^{\mathrm{b}}$ & $4 \mathrm{~h} 16 \mathrm{~min}$ & $13 \mathrm{~h} 20 \mathrm{~min}$ & Morreu \\
\hline
\end{tabular}

a Dose repetida, ${ }^{\mathrm{b}}$ dose única.

Barbosa e Oliveira (2004) atenderam casos de intoxicação por abamectina em bezerros nos municípios de Açailândia, MA, Capitão Poço, PA, e Rondon do Pará, PA.

No surto em Açailândia, MA, de 14 bezerros da raça Nelore, com aproximadamente um mês de idade que foram vermifugados com abamectina, 10 morreram nas primeiras 24 horas após a vermifugação, aparentemente, com a dose preconizada na bula.

No município de Capitão Poço, PA, morreram 10 bezerros recém-nascidos após a administração de abamectina na dose de $1 \mathrm{ml}$ por animal, independentemente do peso, para a prevenção de miíase umbilical.

Em Rondon do Pará, PA, obtivemos históricos em três propriedades. Na primeira a abamectina foi administrada em animais com idades entre oito e 12 meses, sendo 36 bezerros com pesos de $80-150 \mathrm{~kg}$ e em outros 89 , com pesos variando de $180-240 \mathrm{~kg}$. No primeiro lote, constituído por 20 fêmeas e 16 machos, 14 bezerros apresentaram alterações neurológicas, compatíveis com as manifestações relatadas na intoxicação por abamectina, 5 horas após a administração de $5 \mathrm{ml}$ do medicamento, que corresponde a aproximadamente $1,6-3,1$ vezes a dose terapêutica. Os sinais variaram em intensidade, sendo que dois animais que apresentaram sinais mais graves morreram após 12 horas. Nessa mesma propriedade, uma vaca em estado nutricional ruim, de sete anos de idade, com peso de $320 \mathrm{~kg}$ também morreu após a administração de abamectina. Todos os demais melhoraram paulatinamente e, após cinco dias, estavam recuperados.

Numa segunda propriedade, uma novilha de aproximadamente $400 \mathrm{~kg}$ morreu após a vermifugação com $15 \mathrm{ml}$ de abamectina (aprox. 2 vezes a dose terapêutica); além desse animal, adoeceram três bezerros com aproximadamente 8 meses de idade e $180 \mathrm{~kg}$ de peso, os quais apresentaram sinais graves de intoxicação, sendo que dois se recuperaram após duas semanas e um morreu.

Na terceira fazenda, o proprietário relatou intoxicação por abamectina em três bezerros magros, com 4 meses de idade, após receberam a dose preconizada na bula; dois desses animais morreram.

Em 2004, no município de São Mamede, PB, 40 bovinos $(12$ jovens e 28 adultos) receberam abamectina na dose recomendada; 24 horas após a vermifugação todos os animais jovens, com idade de 2-3 meses adoeceram. Os sinais clínicos foram tremores musculares, diminuição dos movimentos proprioceptivos, dificuldade de se manter em estação, protrusão da língua, sonolência e depressão profunda. Dois dos cinco animais que adoeceram gravemente morreram; os outros sete se recuperaram em um período de 15 dias. (Riet-Correa 2006)

Dois episódios de intoxicação por abamectina administrada na dose terapêutica foram observados em bovinos Nelore no

Quadro 2. Alterações clínicas da intoxicação experimental por abamectina em bezerros

\begin{tabular}{|c|c|c|c|c|c|c|c|c|c|c|c|c|c|c|c|c|}
\hline $\begin{array}{c}\text { Animal } \\
\text { no. }\end{array}$ & Paresia & $\begin{array}{l}\text { Hipotonia } \\
\text { muscular }\end{array}$ & $\begin{array}{c}\text { Disme- } \\
\text { tria }\end{array}$ & $\begin{array}{l}\text { Camba- } \\
\text { leio do } \\
\text { corpo }\end{array}$ & $\begin{array}{c}\text { Abdução } \\
\text { exage- } \\
\text { rada }\end{array}$ & $\begin{array}{l}\text { Cruzamen- } \\
\text { to dos } \\
\text { membros }\end{array}$ & $\begin{array}{l}\text { Flexão dos } \\
\text { membros } \\
\text { com apoio } \\
\text { da pinça }\end{array}$ & $\begin{array}{l}\text { Arrastar } \\
\text { da pinça } \\
\text { ao ca- } \\
\text { minhar }\end{array}$ & $\begin{array}{l}\text { Instabi- } \\
\text { lidade }\end{array}$ & Apatia & $\begin{array}{l}\text { Sono- } \\
\text { lência }\end{array}$ & $\begin{array}{c}\text { Tendência } \\
\text { ao decú- } \\
\text { bito }\end{array}$ & $\begin{array}{l}\text { Permanên- } \\
\text { cia ao de- } \\
\text { cúbito } \\
\text { lateral }\end{array}$ & $\begin{array}{l}\text { Instabili- } \\
\text { dade da } \\
\text { cabeça }\end{array}$ & $\begin{array}{c}\text { Apoio } \\
\text { da cabe- } \\
\text { ça no } \\
\text { solo }\end{array}$ & $\begin{array}{l}\text { Movimen- } \\
\text { tos de la- } \\
\text { teralidade } \\
\text { da cabeça }\end{array}$ \\
\hline 1346 & $-a$ & - & + & +++ & +++ & + & + & + & + & +++ & - & $\mathrm{CONS}^{\mathrm{b}}$ & - & - & - & - \\
\hline 1344 & +++ & +++ & ++ & ++ & +++ & ++ & ++ & +++ & +++ & +++ & +++ & CONS & FRQ & +++ & FRQ & +++ \\
\hline 1340 & ++ & ++ & ++ & ++ & ++ & + & - & + & ++ & ++ & +++ & CONS & - & - & - & - \\
\hline 1342 & +++ & +++ & + & ++ & +++ & ++ & +++ & - & ++ & ++ & + & FRQ & FRQ & +++ & FRQ & + \\
\hline 1325 & +++ & ++ & + & +++ & ++ & - & - & - & +++ & +++ & + & FRQ & $\mathrm{OC}$ & +++ & FRQ & ++ \\
\hline 1347 & +++ & +++ & + & +++ & + & + & - & - & +++ & +++ & +++ & CONS & FRQ & +++ & FRQ & - \\
\hline 1343 & +++ & +++ & ++ & ++ & ++ & - & - & - & + & +++ & + & CONS & FRQ & - & OC & - \\
\hline 1320 & +++ & +++ & - & +++ & ++ & - & +++ & - & ++ & +++ & +++ & CONS & FRQ & +++ & FRQ & + \\
\hline 1524 & +++ & +++ & + & ++ & +++ & ++ & +++ & +++ & +++ & +++ & +++ & CONS & - & +++ & FRQ & +++ \\
\hline
\end{tabular}

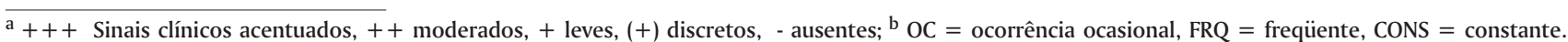


Quadro 2 (Cont.). Alterações clínicas da intoxicação iatrogênica por abamectina em bezerros

\begin{tabular}{|c|c|c|c|c|c|c|c|c|c|c|c|c|c|c|}
\hline $\begin{array}{c}\text { Animal } \\
\text { no. }\end{array}$ & $\begin{array}{c}\text { Desequi- } \\
\text { líbrio }\end{array}$ & $\begin{array}{l}\text { Tendência } \\
\text { a quedas }\end{array}$ & $\begin{array}{c}\text { Fascicula- } \\
\text { ções mus- } \\
\text { culares in- } \\
\text { termiten- } \\
\text { tes e gene- } \\
\text { ralizadas }\end{array}$ & $\begin{array}{l}\text { Sensibilida- } \\
\text { de superfi- } \\
\text { cial (por pi- } \\
\text { cadas com } \\
\text { agulha } \\
\text { fina) }\end{array}$ & $\begin{array}{l}\text { Respos- } \\
\text { ta à dor } \\
\text { profun- } \\
\text { da (pin- } \\
\text { çamen- } \\
\text { to) }\end{array}$ & $\begin{array}{l}\text { Hiper- } \\
\text { estesia }\end{array}$ & $\begin{array}{l}\text { Sensibi- } \\
\text { lidade } \\
\text { da face }\end{array}$ & Dispnéia & $\begin{array}{c}\text { Reflexo } \\
\text { de } \\
\text { sucção }\end{array}$ & Disfagia & $\begin{array}{c}\text { Saliva- } \\
\text { ção }\end{array}$ & $\begin{array}{c}\text { Paresia } \\
\text { da lín- } \\
\text { gua }\end{array}$ & $\begin{array}{l}\text { Reflexo } \\
\text { pupilar }\end{array}$ & $\begin{array}{l}\text { Lacrime- } \\
\text { jamento }\end{array}$ \\
\hline 1346 & $+++^{a}$ & +++ & - & $\mathrm{N}^{\mathrm{b}}$ & $\mathrm{N}$ & ++ & $\mathrm{N}$ & $(+)$ & $\mathrm{N}$ & - & $(+)$ & +++ & AUS & $(+)$ \\
\hline 1344 & +++ & +++ & $++^{b}$ & $\begin{array}{c}\text { RET } \\
+++\end{array}$ & $\begin{array}{c}\text { RET } \\
+++\end{array}$ & +++ & $\begin{array}{c}\text { RET } \\
+++\end{array}$ & +++ & $\begin{array}{c}\text { RET } \\
+\end{array}$ & - & +++ & + & AUS & +++ \\
\hline 1340 & ++ & ++ & - & $\mathrm{N}$ & $\mathrm{N}$ & - & $\mathrm{N}$ & - & $\mathrm{N}$ & - & - & - & $\mathrm{N}$ & - \\
\hline 1342 & +++ & +++ & - & $\mathrm{N}$ & $\mathrm{N}$ & - & $\mathrm{N}$ & - & $\mathrm{N}$ & - & +++ & +++ & $\mathrm{N}$ & - \\
\hline 1325 & +++ & +++ & + & $\begin{array}{c}\text { RET } \\
+++\end{array}$ & AUS & ++ & $\mathrm{N}$ & + & $\begin{array}{c}\text { RET } \\
+++\end{array}$ & +++ & ++ & +++ & $\mathrm{N}$ & - \\
\hline 1347 & +++ & +++ & ++ & $\mathrm{N}$ & $\mathrm{N}$ & - & $\mathrm{N}$ & +++ & $\mathrm{N}$ & - & +++ & +++ & AUS & +++ \\
\hline 1343 & +++ & +++ & +++ & $\begin{array}{c}\text { RET } \\
+++\end{array}$ & AUS & - & $\begin{array}{c}\text { RET } \\
+\end{array}$ & +++ & AUS & ++ & +++ & +++ & AUS & +++ \\
\hline 1320 & ++ & ++ & ++ & $\begin{array}{c}\text { RET } \\
+++\end{array}$ & AUS & ++ & $\mathrm{N}$ & +++ & AUS & - & - & +++ & $\mathrm{N}$ & - \\
\hline 1524 & +++ & +++ & +++ & $\begin{array}{l}\text { AUM } \\
+++\end{array}$ & $\begin{array}{l}\text { AUM } \\
+++\end{array}$ & +++ & $\mathrm{N}$ & - & $\begin{array}{l}\text { RET } \\
++\end{array}$ & + & +++ & +++ & AUS & - \\
\hline
\end{tabular}

${ }^{\mathrm{a}}+++$ Sinais clínicos acentuados, ++ moderados, + leves, $(+)$ discretos, - ausentes; ${ }^{\mathrm{b}} \mathrm{N}=$ resposta normal, $\mathrm{RET}=$ retardada, $\mathrm{AUM}=$ aumentada, $\mathrm{AUS}=$ ausente
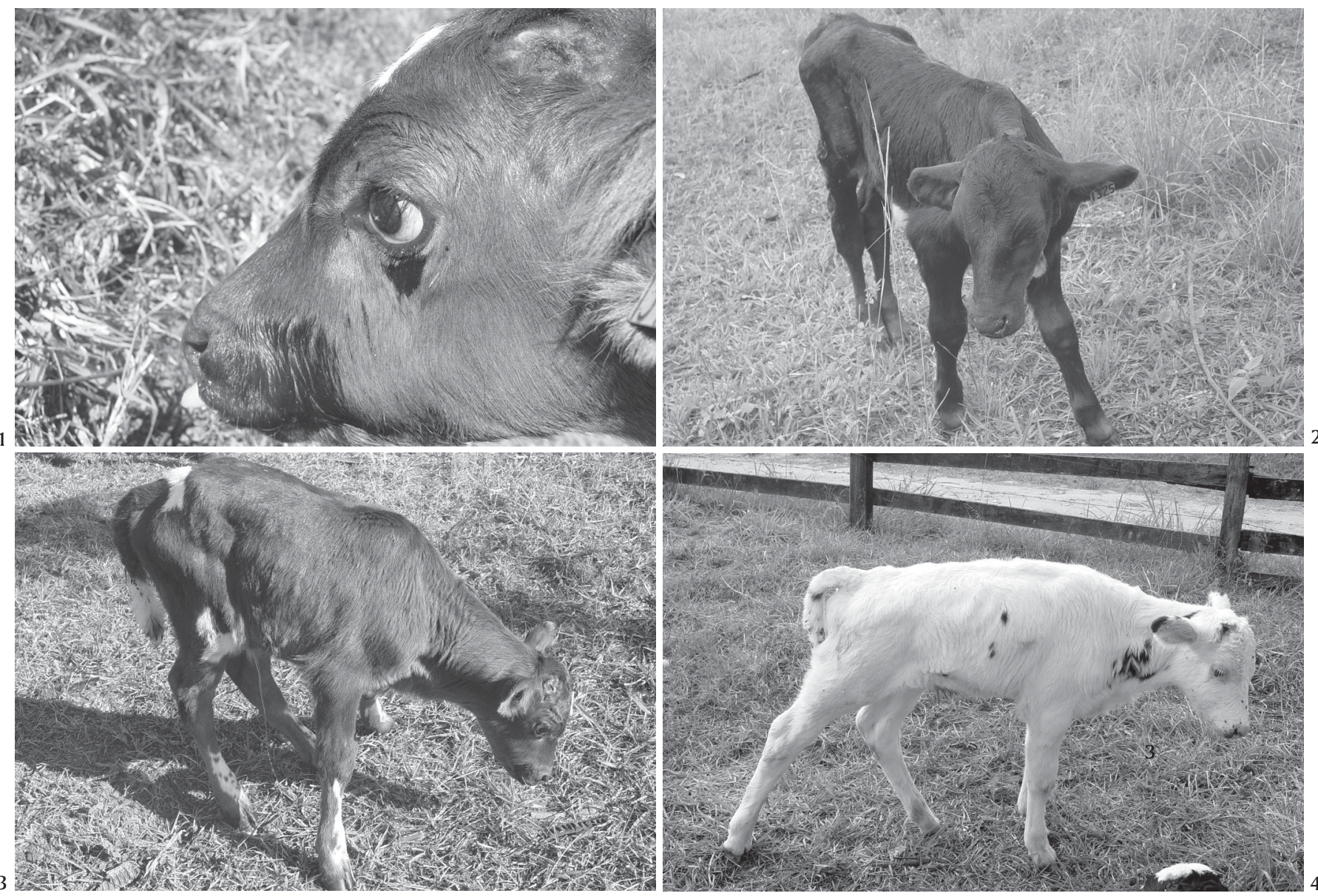

Fig.1.Lacrimejamento, protusão da língua e salivação(Bezerro 1347, recebeu 6 doses terapêuticas de abamectina em uma única administração), na intoxicação experimental por abamectina.

Fig.3. Abdução exagerada dos membros torácicos, posicionamento atípico desequilibrado dos membros pélvicos e manutenção da cabeça para baixo (Bezerro 1320, recebeu 10 doses terapêuticas de abamectina em uma única administração).

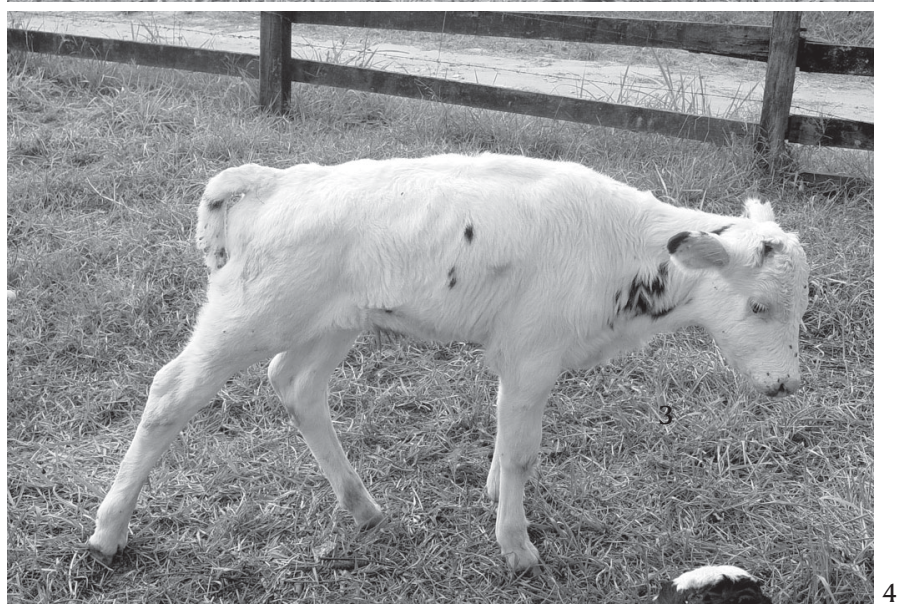

Fig.2. Abdução exagerada dos membros torácicos e cruzamento dos membros pélvicos (Bezerro 1325, recebeu 5 doses terapêuticas de abamectina em uma única administração).

Fig.4. Acentuada rigidez dos membros - andar hipermétrico (Bezerro 1344, recebeu 1 dose terapêutica diária de abamectina por 11 dias). 
município de Corumbá, MS. O início dos sinais clínicos variou de seis a 12 horas e entre as principais manifestações observaramse incoordenação, língua protrusa, posicionamento anormal da cabeça e orelhas caídas. Dos 47 animais que adoeceram, cerca de 15 se recuperaram em aproximadamente uma semana e 32 morreram ( 6 vacas, 13 bezerros jovens e 13 bezerros desmamados há 40 dias) de 20-48 horas após a observação dos primeiros sinais. (Wouters 2005)

No município de São Nicolau, RS, observou-se que, após a administração de abamectina na dose recomendada em 137 animais, 15 bovinos adoeceram e, destes, sete morreram em cerca de 48 horas, sendo quatro vacas, duas novilhas e um touro.(Wouters 2005)

Nos animais das diferentes propriedades, que foram necrospiados, nenhuma alteração macroscópica ou microscópica foi observada no SNC ou em qualquer outro órgão.

\section{Casos experimentais}

Os sinais clínicos observados nos animais intoxicados caracterizaram-se por disfunções neurológicas, em que houve uma fase inicial de hiperexcitabilidade, seguida por hipotonia muscular generalizada e depressão progressiva (Quadro 1 e 2, Fig.1-4).

Dos nove animais que foram submetidos à administração de abamectina, cinco morreram. Não foram observadas alterações macro ou microscópicas dignas de notas.

\section{DISCUSSÃO}

A ocorrência de diversos surtos, totalizando pelo menos 57 mortes em apenas 4 anos, relatados somente por três serviços de diagnósticos contactados, indica que a intoxicação por abamectina seja freqüente e que, provavelmente, o número de casos de intoxicação iatrogênica vem sendo subestimado, visto que os sinais podem ser leves, transitórios e que muitos animais apresentam rápida recuperação.

Durante a vermifugação, os erros não são incomuns e podem ocorrer intoxicações quando as doses são "estimadas" ou mesmo "padronizadas", para facilitar o manejo de animais de diferentes pesos pertencentes a um mesmo lote ou rebanho; assim, quando não são respeitadas as restrições referentes aos animais mais sensíveis, qualquer animal, em tese, pode se intoxicar pelas avermectinas.

Benz \& Cox (1989) e Courtney \& Roberson (1995) consideram a margem de segurança das avermectinas ampla e mesmo para animais altamente susceptíveis à intoxicação, como os bezerros com menos de 4 meses, a dose de até 5 vezes superior a recomendada não seria letal, muito embora, tenham observado que, em geral, sinais severos de intoxicação e mortes ocorrem quando a abamectina é utilizada em doses muito acima das recomendadas (5-10 vezes superior) ou quando é administrada a animais que apresentam uma maior sensibilidade. Contudo, no Brasil, ao que tudo indica, muitos casos de intoxicação iatrogênica ocorreram após a administração da dose terapêutica ou envolveram animais com idade acima daquela em que há restrições. Em nossos experimentos, sinais leves a severos de toxicidade foram evidenciados em todos os animais que receberam abamectina, até naqueles em que o medicamento foi administrado na dose terapêutica (em dose única) e na metade da dose recomendada (em doses repetidas).

Em muitos casos de intoxicação iatrogênica não é possível obter informações exatas sobre a idade dos animais, doses administradas, bem como sobre as características clínicas, porém, em algumas das comunicações referidas, $o$ início dos sinais clínicos variou de 6-12h e o período de evolução, de 12-48h nos casos em que o desfecho culminou com a morte e de no máximo 7 dias até a completa recuperação clínica.

Experimentalmente houve grande variação entre o período de administração e o início dos sinais (de 50min a 10h24min), bem como na evolução (de 13h20min a 21 dias), embora essa oscilação, em parte, possa ser creditada à grande diferença entre as dosagens. Mesmo assim, os Bezerros 1342 e 1325, que receberam 5 vezes a dose terapêutica de abamectina, apresentaram marcada variação no início dos sintomas após a administração e na evolução clínica, o que poderia ser atribuído à diferenças na resistência individual à droga.

Os animais intoxicados pelas avermectinas podem apresentar manifestações clínicas de natureza variada e inespecífica, visto que receptores GABAérgicos encontram-se em praticamente todos os neurônios do SNC e que são responsáveis por regular o tônus basal neural do cérebro (Turner \& Schaeffer 1989). Nesse estudo, o quadro clínico inicial observado, em alguns animais manifestou-se por uma fase de excitação transitória, de inquietação e hiperestesia, manifestada pelo aumento na intensidade da resposta aos reflexos panicular, patelar, de beliscamento e através de picadas por agulha fina, seguido de depressão e coma, $o$ que corresponde ao descrito por Payne \& Soderlund (1993) em ratos intoxicados por abamectina e ivermectina.

Na maioria dos animais, as manifestações descritas como as mais freqüientes, provavelmente são resultantes da ação inibitória do GABA e, conseqüentemente, de uma diminuição do tônus muscular, a qual caracteriza uma fase intermediária. Sendo assim, algumas das alterações posturais e motoras podem ser somente uma manifestação da hipotonia muscular generalizada. Finalmente, desenvolve-se um quadro terminal de depressão acentuada, representada por tendência ao decúbito, sonolência e apatia progressiva. Embora o GABA seja um neurotransmissor inibitório, as alterações clínicas iniciais, podem ser explicadas pela excitação celular exercida através da estimulação de efluxo clorídrico pelos receptores insensíveis ao GABA, que funcionam como antagonistas desse neurotransmissor e resultam em uma fase hiperexcitatória. Porém, a depressão, que é observada numa fase subseqüiente, se estabelece à medida que há hiperpolarização secundária à estimulação do GABA, segundo a abordagem de Payne \& Soderlund (1993).

Independente do neurotransmissor envolvido na potencialização e/ou ativação dos canais de cloro nos parasitas, ser glutamato ou GABA (Courtney \& Roberson 1995, Corwin 1999, Nobmann et al. 2001, Taylor 2001, Andrade \& Santarém 2002), as manifestações, que são caracterizadas principalmente por depressão e distúrbios proprioceptivos, são condizentes com a ação inibitória provocada por medicamentos que funcionam como agonistas do GABA. Portanto, é provável que o glutamato exerça efeito apenas sobre os parasitas, pois nos mamíferos sua ação é excitatória e não corresponde às manifestações observadas em nossos experimentos, nem com as descritas até o presente na literatura. 
Há poucas lesões anátomo-patológicas descritas nos casos de intoxicações agudas pelas avermectinas e nenhuma alteração microscópica relacionada com as manifestações neurológicas observadas, as quais constituem as principais alterações clínicas nos casos de intoxicação por avermectinas em bezerros.

Técnicas de imuno-histoquímica que utilizam anticorpos seletivos contra a glutamato descarboxilase, presente apenas em neurônios sintetizadores do GABA, têm sido usadas para o mapeamento das vias GABAérgicas no SNC (Silva 2002). Sendo assim, poderia ser considerada a possibilidade do uso de anticorpos, especialmente contra as avermectinas na tentativa de se mapear os locais de ligação ou de maior afinidade entre as células nervosas e o medicamento, já que nas colorações de rotina (HE) não se têm observado lesões histopatológicas. Em virtude da alta complexidade dos vários receptores do GABA e de suas interações funcionais, aliada à ausência de alterações macro e microscópicas consistentes, existentes na literatura e descritas no presente experimento, a patogenia da intoxicação pela abamectina não está bem esclarecida e necessita de estudos bioquímicos e neurológicos mais aprofundados.

Agradecimentos.- Ao professor Carlos Hubinger Tokarnia pela ajuda nos trabalhos experimentais; aos professores José Diomedes Barbosa Neto, David Driemeier, Franklin Riet-Correa, Flademir Wouters e Carlos Magno Oliveira pelo envio dos históricos de casos iatrogênicos; aos Médicos Veterinários Douglas Marques Macedo e Carlos Torres Ribeiro pela participação nos experimentos; à Pesagro, Estação Experimental de Seropédica, Área de Bovino de Leite, por ceder os animais utilizados nos experimentos.

\section{REFERÊNCIAS}

Andrade S.F. \& Santarém V.A. 2002. Endoparasiticida e ectoparasiticida, p.469-470. In: Andrade S.F. (ed.) Manual Terapêutica Veterinária. $2^{\text {a }}$ ed. Roca, SP.

Ayres M.C.C. \& Almeida M.A.O. 2002. Agentes antinematódeos, Agentes antiparasitários, p.476-488. In: Spinosa H.S., Górniak S.L. \& Bernadi M.M. (ed.) Farmacologia Aplicada à Medicina Veterinária. $3^{\mathrm{a}}$ ed. Guanabara Koogan, Rio de Janeiro.

Barbosa J.D.N. 2004. Comunicação pessoal (Clínica de Animais de Grande Porte, Universidade Federal do Pará, PA).

Barragry T.B. 1994. Clinical pharmacology of endoparasiticides, p.104110. In: Ibid. (ed.) Veterinary Drug Therapy. Lea \& Febiger, Philadelphia.

Basílio J. 2005. A revolucionária descoberta. Disponível em: $<$ http:// www.vaquejadas.com./index.php?fa.mat.infmateria\&mat_id $=1168>$. Acesso em: 08 fev. 2005.

Benz G.W. \& Cox J.L. 1989. Use of abamectin in cattle, p.230-233. In: Campbell W.C. (ed.) Ivermectin and Abamectin. Springer Verlag, New York.

Burg R.W., Miller B.M., Baker E.E., Birnbaum J., Currie S.A., Hartman R., Kong Y.L., Monaghan R.L., Olson G., Putter I., Tunac J.B., Wallick H., Stapley E.O., Oiwa R. \& Omura S. 1979. Avermectins, new family of potent anthelmintic agents: producting organism and fermentation. Antimicrob Agents Chemother. 15:361-367.

Button C., Barton R., Honey P. \& Rickford P. 1988. Avermectin toxicity in calves and an evaluation of picrotoxin as an antidote. Aust. Vet. J. 65:157158. (Cit. Pulliam \& Preston 1989)

Chabala J.C., Mrozik H., Tolman R.L. Eskola P., Lusi A., Peterson L.H.,
Woods M. F. \& Fisher M.H. 1980. Ivermectin, a new broad-spectrum antiparasitic agent. J. Med. Chemist. 23:1134-1336.

Corwin R. M. 1999. Special therapy, Anthelmintic therapy, p.34-35. In: Howard J.M. \& Smith R.A. (ed.) Current Veterinary Therapy, Food Animal Pratice. 4th ed. W.B. Saunders, Philadelphia.

Courtney C.H. \& Roberson E.L. 1995. Chemotherapy of parasitic disease. Antinematodal drugs, p.916-922. In: Adams H.R. (ed.) Veterinary Pharmacology and Therapeutics. 7th ed. Iowa State University Press, Ames.

Driemeier D. 2001. Comunicação pessoal (Departamento de Patologia Clínica Veterinária, Setor de Patologia Veterinária, Universidade Federal do Rio Grande do Sul, Porto Alegre, RS).

Fisher M.H. \& Mrozik H. 1989. Chemistry, p.1-23. In: Campbell W.C. (ed.) Ivermectin and Abamectin. Springer Verlag, New York.

Forbes A.B. 1993. A review of regional and temporal use of avermectinas in cattle and horses worldwide. Vet. Parasitol. 48:19-28.

Lankas G.R. \& Gordon L.R. 1989. Toxicology, p.89-112. In: Campbell W.C. (ed.) Ivermectin and Abamectin. Springer Verlag, New York.

Lankas G.R., Cartwright M.E. \& Umbenhauer D. 1997. P-glicoprotein deficiency in a subpopulação of CF-1 mice enhances avermectin-induced neurotoxicity. Toxicol. Appl. Pharmacol. 143:357-365.

Moore L.F. 1999. Dermatologic disease. External parasites, p.731. In: Corviw R.M. (ed.) Current Veterinary Therapy, Food Animal Pratice. 4th ed. W.B. Saunders, Philadelphia.

Nobmann S., Bauer B. \& Fricker G. 2001. Ivermectin excretion by isolates functionally intact brain endothelial capillaries. Brit. J. Pharmacol. 132:722-728.

Oliveira C.M. 2004. Comunicação pessoal (Clínica de Animais de Grande Porte, Universidade Federal do Pará, Castanhal, PA).

Payne G.T. \& Soderlund D.M. 1993. Actions os avermectina analogues on ã-aminobutyric acid (GABA)-sensitive and GABA-insensitive chloride channels in mouse brain. Pestic. Bioch. Physiol. 47:178-184.

Pulliam J.D. \& Preston J.M. 1989. Safety of ivermectin in target animals, p.149-161. In: Campbell W.C. (ed.) Ivermectin and Abamectin. Springer Verlag, New York.

Radostits O.M., Gay C.C., Blood D.C. \& Hinchcliff K.W. 2002. Doenças causadas por substâncias químicas inorgânicas e produtos químicos utilizados nas fazendas, p.1417-1471. In: Ibid. (ed.) Clínica Veterinária: um tratado de doenças dos bovinos, ovinos, suínos, caprinos e eqüinos. $9^{\mathrm{a}}$ ed. Guanabara Koogan, Rio de Janeiro.

Riet-Correa F. 2006. Comunicação pessoal (Hospital Veterinário do Centro de Saúde e Tecnologia Rural, Universidade Federal de Campina Grande, Patos, PB).

Silva E.C. 2002. Neurotransmissão aminérgica central, p.287-301. In: Silva P. (ed.) Farmacologia. $6^{\text {a }}$ ed. Guanabara Koogan, Rio de Janeiro.

Steel J.W. 1993. Pharmacokinetics and metabolism of avermectins in livestock. Vet. Parasitol. 48:45-57.

Taylor M.A. 2001. Review: recent developments in ectoparasites. Vet. J. 161:253-268.

Tracy J.M. \& Webster L. T. Jr. 2001. Drugs used in the chemotherapy of helminthiasis, p.1131-1133. In: Harman J.G., Limbird L.E \& Gilman G.A. (ed.) Goodman \& Gilman's The Pharmacological Basis of Therapeutics. 10th ed. Internacional Edition, New York.

Turner M.J. \& Schaeffer J.M. 1989. Mode of action of ivermectin, p.73-88. In: Campbell W.C. (ed.) Ivermectin and Abamectin. Springer Verlag, New York.

Wouters F. 2005. Comunicação pessoal (Departamento de Medicina Veterinária, Universidade Federal de Lavras, MG). 